$$
\begin{aligned}
& \nu \quad=\text { kinematic viscosity of fluid } \\
& \rho \quad=\text { density of fluid } \\
& \tau \quad=\text { shear stress } \\
& \tau_{w}=\text { shear stress at wall } \\
& \langle\text { Subscripts }\rangle \\
& o \quad=\text { refers to inlet of porous pipe } \\
& L \quad=\text { refers to outlet of porous pipe } \\
& 0 \quad=\text { refers to no mass flux at wall }
\end{aligned}
$$

\section{Literature cited}

1) Aureille, R. and A. Favre, : Publ. Sci. et Tech. Ministère Air (1967)

2) Bird, R. B., W. E. Stewart, and E. N. Lightfoot,

"Transport Phenomena", John Wiley and Sons, Inc., $\left[\mathrm{cm}^{2} / \mathrm{sec}\right]$

$\left[\mathrm{g} / \mathrm{cm}^{3}\right]$

$\left[\mathrm{g} / \mathrm{cm} \cdot \mathrm{sec}^{2}\right]$

$\left[\mathrm{g} / \mathrm{cm} \cdot \mathrm{sec}^{2}\right]$ 
the temperature in solid particles satisfies the equation of conduction of heat.

\section{Inducement of Basic Equations}

By considering the transfer of heat to an element of fluid, in a similar way to Schumann's, we obtain

$$
C_{G} \rho_{G} F_{e} \frac{\partial t_{G}}{\partial \theta}+C_{G} G \frac{\partial t_{G}}{\partial Z}=h a_{V}\left(t_{S}(a)-t_{G}\right)
$$

Eq. (1) is similar to one of Schumann's equations except that solid temperature is replaced by surface temperature. Schumann did not distinguish between the surface temperature of solid and the temperature of solid assuming uniform temperature of solid.

By introducing a new independent variable

$$
\theta^{\prime}=\theta-Z / u
$$

where

$$
u=G /\left(\rho_{G} F_{e}\right)
$$

and

$$
k_{2}=h a_{V} /\left(C_{G} \rho_{G} F_{e}\right)
$$

Eq. (1) is reduced to

$$
u \frac{\partial t_{G}}{\partial Z}=-k_{2}\left(t_{G}-t_{S}(a)\right)
$$

Schumann also introduced another equation by considering an element of the solid assuming uniform temperature in solid particles:

$$
\frac{\partial t_{S}}{\partial \theta^{\prime}}=k_{1}\left(t_{G}-t_{S}\right)
$$

where

$$
k_{1}=\frac{h a_{V}}{C_{S} \rho_{S}\left(1-F_{e}\right)}
$$

This equation is very simple but limits its usefulness owing to its assumption.

In this paper, instead of this Schumann's equation, the solution of the equation of heat conduction where the initial temperature is zero, and radiation takes place at its surface into a medium is introduced by means of Duhamel's theorem ${ }^{2}$. This equation, although the solution is originally provided for obtaining solid temperature on the condition that gas temperature is given as a prescribed function of time, is considered as a partial integral equation on unknown dependent variables of gas and solid temperature, and independent variables of time and distance from the inlet of gas.

The equation is

$$
\begin{aligned}
t_{S}(r)= & \frac{2 h^{\prime} \kappa}{r} \sum_{n=1}^{\infty} e^{-\kappa \alpha_{n}^{2} \theta^{\prime}} \frac{\left(a^{2} \alpha_{n}^{2}+\left(a h^{\prime}-1\right)^{2}\right)}{\left(a^{2} \alpha_{n}^{2}+a h^{\prime}\left(a h^{\prime}-1\right)\right)} \\
& \times \sin \left(a \alpha_{n}\right) \sin \left(r \alpha_{n}\right) \int_{0}^{\theta^{\prime}} e^{\kappa \alpha_{n}^{2} \lambda} t_{G} d \lambda
\end{aligned}
$$

where $\alpha_{n}$ are the positive root of

$$
(a \alpha) \cot (a \alpha)+a h^{\prime}-1=0
$$

Eq. (8) is modified to

$$
\begin{aligned}
& t_{S}(r)=t_{G} \\
& -\left(2 a h^{\prime}\right) \sum_{n=1}^{\infty} \frac{\sin \left(a \alpha_{n}\right)}{\left(a \alpha_{n}\right)} \frac{\sin \left(r \alpha_{n}\right)}{\left(r \alpha_{n}\right)} \frac{\left(a^{2} \alpha_{n}^{2}+\left(a h^{\prime}-1\right)^{2}\right)}{\left(a^{2} \alpha_{n}^{2}+a h^{\prime}\left(a h^{\prime}-1\right)\right)}
\end{aligned}
$$

$$
\times\left(t_{G}-\kappa \alpha_{n}^{2} e^{-\kappa \alpha_{n}^{2} \theta^{\prime}} \int_{0}^{\theta^{\prime}} e^{\kappa \alpha_{n}^{2} \lambda} t_{G} d \lambda\right)
$$

Substituting $r=a$ in Eq.(10), we have

$$
\begin{aligned}
t_{S}(a)=t_{G} & -\left(2 a h^{\prime}\right) \sum_{n=1}^{\infty} \frac{\sin ^{2}\left(a \alpha_{n}\right)}{\left(a \alpha_{n}\right)^{2}} \frac{\left(a^{2} \alpha_{n}^{2}+\left(a h^{\prime}-1\right)^{2}\right)}{\left(a^{2} \alpha_{n}^{2}+a h^{\prime}\left(a h^{\prime}-1\right)\right)} \\
& \times\left(t_{G}-\kappa \alpha_{n}^{2} e^{-\kappa \alpha_{n}^{2} \theta^{\prime}} \int_{0}^{\theta^{\prime}} e^{\kappa \alpha_{n}^{2} \lambda} t_{G} d \lambda\right)
\end{aligned}
$$

Eq. $(10)^{\prime}$ and (5) are two simultaneous partial integro-differential equations for gas temperature $t_{G}$ and solid surface temperature $t_{S}(a)$ in the independent variables of $\theta^{\prime}$ and $Z$.

These are the basic equations which determine the transfer of heat in a packed bed in this problem.

Boundary condition for the inlet gas gives

$$
t_{a}=t_{0}, Z=0
$$

Consider that the temperature of solid is at zero until the gas arrives at the position of the solid from the inlet.

$$
t_{S}(r)=0, \quad \theta^{\prime}=\theta-Z / u=0
$$

\section{Solution of the Equations}

\section{1) Approximate analytical solution}

Taking the first term of the infinite series of Eq. (10) the approximate equation is given as:

$$
\begin{aligned}
t_{S}(a)=t_{G} & -\left(2 a h^{\prime}\right) \frac{\sin ^{2}\left(a \alpha_{1}\right)}{\left(a \alpha_{1}\right)^{2}} \frac{\left(a^{2} \alpha_{1}^{2}+\left(a h^{\prime}-1\right)^{2}\right)}{\left(a^{2} \alpha_{1}^{2}+a h^{\prime}\left(a h^{\prime}-1\right)\right)} \\
& \times\left(t_{G}-\kappa \alpha_{1}^{2} e^{-\kappa \alpha_{1}^{2} \theta^{\prime}} \int_{0}^{\theta^{\prime}} e^{\kappa \alpha_{1}^{2} \lambda} t_{G} d \lambda\right)
\end{aligned}
$$

Substituting it into Eq. (5) gives

$$
\begin{aligned}
u \frac{\partial t_{G}}{\partial Z}= & -\left(2 a h^{\prime}\right) k_{2} \frac{\sin ^{2}\left(a \alpha_{1}\right)}{\left(a \alpha_{1}\right)^{2}} \frac{\left(a^{2} \alpha_{1}^{2}+\left(a h^{\prime}-1\right)^{2}\right)}{\left(a^{2} \alpha_{1}^{2}+a h^{\prime}\left(a h^{\prime}-1\right)\right)} \\
& \times\left(t_{G}-\kappa \alpha_{1}^{2} e^{-\kappa \alpha_{1}^{2} \theta^{\prime}} \int_{0}^{\theta^{\prime}} e^{\kappa \alpha_{1}^{2} \lambda} t_{G} d \lambda\right)
\end{aligned}
$$

By introducing two new independent variables

$$
\begin{aligned}
& \tau=\left(\kappa \alpha_{1}^{2}\right) \theta^{\prime} \\
& \xi=\left(\varepsilon k_{2} / u\right) Z
\end{aligned}
$$

where

$$
\varepsilon=\left(2 a h^{\prime}\right) \frac{\sin ^{2}\left(a \alpha_{1}\right)^{2}}{\left(a \alpha_{1}\right)^{2}} \frac{\left(a^{2} \alpha_{1}^{2}+\left(a h^{\prime}-1\right)^{2}\right)}{\left(a^{2} \alpha_{1}^{2}+a h^{\prime}\left(a h^{\prime}-1\right)\right)}
$$

Eq. (14) is reduced to

$$
\frac{\partial t_{G}}{\partial \xi}=-t_{G}+e^{-\tau} \int_{0}^{\tau} e^{\lambda} t_{G} d \lambda
$$

By application of Laplace transform, the solution of Eq.(18) satisfying the boundary condition of Eqs. (11) and (12) is given by (See Appendix)

$$
t_{G} / t_{0}=e^{-(\xi+\tau)} I_{0}(2 \sqrt{\xi \tau})+\int_{0}^{\tau} e^{-(\xi+2)} I_{0}(2 \sqrt{\xi \lambda}) d \lambda
$$

Eq.(5) is also reduced to simpler form, and the surface temperature of solid is given by

$$
t_{S}(a) / t_{0}=t_{G} / t_{0}-\varepsilon e^{-\xi-\tau} I_{0}(2 \sqrt{\xi \tau})
$$

Eq. (19) has the same form as Furnas' analytical solution in spite of the different definition of the variables, so that we can use the numerical chart given by Schumann ${ }^{9)}$ and Furnas ${ }^{4}$ for calculation.

In the particular case where the thermal diffusivity of the solid becomes infinite, we have 


$$
\begin{aligned}
& \lim _{\kappa \rightarrow \infty} \kappa \alpha_{1}^{2}=\frac{h a_{V}}{C_{S} \rho_{S}\left(1-F_{e}\right)} \\
& \lim _{\kappa \rightarrow \infty} \varepsilon=1
\end{aligned}
$$

Using these results, Eqs.(15) and (16) become

$$
\begin{aligned}
\tau & =\frac{h a_{V}}{C_{S} \rho_{S}\left(1-F_{e}\right)} \theta^{\prime} \\
\xi & =\frac{h a_{V}}{G C_{G}} Z
\end{aligned}
$$

These are identical with the variables defined by Schumann and Furnas, and not only Eq. (19) but also Eq. $(20)$ is identical with that of Furnas.

Using the results of Eqs. (21) and (22), it is easily proved mathematically that Eq. $(10)^{\prime}$ is reduced to another of Schumann's equations (See Appendix).

Substituting the solution(19) into $\mathrm{Eq} \cdot(10)^{\prime}$, we have the $n$-th term as follows:

$$
\begin{aligned}
& A_{n}=t_{0}\left(2 a h^{\prime}\right) \frac{\sin ^{2}\left(a \alpha_{n}\right)}{\left(a \alpha_{n}\right)^{2}} \frac{\left(a^{2} \alpha_{n}^{2}+\left(a h^{\prime}-1\right)^{2}\right)}{\left(a^{2} \alpha_{n}^{2}+a h^{\prime}\left(a h^{\prime}-1\right)\right)} e^{-\xi} \\
& \times e^{-\left(\alpha_{n}^{2} / \alpha_{1}^{2}\right) \tau}\left(\int_{0}^{\tau} e^{\left(\alpha_{n}^{2} / \alpha_{1}^{2}-1\right) \lambda} \sqrt{\frac{\xi}{\lambda}} I_{1}(2 \sqrt{\xi \lambda}) d \lambda+1\right)
\end{aligned}
$$

Let us evaluate the possible error by neglecting terms after the first in Eq. $(10)^{\prime}$ using the inequalities

$$
\alpha_{n}>-\frac{\pi}{2}+(n-1), \quad n>2
$$

and

$$
e^{-\hat{\xi}-\lambda} \sqrt{\frac{\xi}{\lambda}} I_{1}(2 \sqrt{\xi \lambda}) \leqq e^{-1}
$$

We may evaluate the error

$$
\begin{gathered}
R=\left|\sum_{n=2}^{\infty} A_{n}\right| \leqq \sum_{n=2}^{\infty}\left|A_{n}\right| \\
<t_{0}(2 a h)^{\prime}\left(8.9 \times 10^{-4}\left(a \alpha_{1}\right)^{2}+0.095 e^{-\left(\alpha_{2} / \alpha_{1}\right)^{2}}\right)
\end{gathered}
$$

For example, assuming $a h^{\prime}=4.0$, we have

$$
R<\left(4.29 \times 10^{-2}+0.76 e^{-4.45 t}\right) t_{0}
$$

The center temperature of the solid may be estimated by substituting the solution (19) into Eq. (10) and computing to some arbitrary term of the series using the following relationships:

$$
\lim _{r \rightarrow 0} \frac{\sin \left(r \alpha_{n}\right)}{\left(r \alpha_{n}\right)}=1.0
$$

\section{2) Precise numerical solution}

By introducing the dimensionless variables of Eq. (15), Eq.(16) and Eq.(17), Eq.(6) and Eq.(10)' are reduced to

$$
\begin{gathered}
\frac{\partial t_{G}}{\partial \xi}=-\frac{1}{\varepsilon}\left(t_{G}-t_{S}(a)\right) \\
t_{S}(a)=t_{G}-\left(2 a h^{\prime}\right) \sum_{n=1}^{\infty} \frac{\sin ^{2} \beta_{n}}{\beta_{n}^{2}} \frac{\left(\beta_{n}^{2}+\left(a h^{\prime}-1\right)^{2}\right)}{\left(\beta_{n}^{2}+a h^{\prime}\left(a h^{\prime}-1\right)\right)} \\
\times\left(t_{G}-\frac{\beta_{n}^{2}}{\beta_{n}^{2}} e^{-\left(\beta_{n}^{2} / \beta_{1}^{2}\right) \tau} \int_{0}^{\tau} e^{\left(\beta_{n}^{2} / \beta_{1}^{2}\right) \lambda} t_{G} d \lambda\right)
\end{gathered}
$$

where $\beta_{n}=a \alpha_{n}$

Let the range in $\xi$ be divided into equal intervals $\Delta \xi$, and $\tau$ into intervals $\Delta \tau$, and $t_{G(i)}$ and $t_{S(i)}$ denote the temperature of the gas and the solid at the point $i \Delta \xi$ at $\tau=l \Delta \tau$. Then from Eq. (30) we have the recurrence formula on the position

$$
t_{G(i)}=t_{G(i-1)} e^{-\Delta \xi / \varepsilon}+0.5\left(t_{S(i-1)}(a)+t_{S(i)}(a)\right)\left(1-e^{-\Delta \xi / \varepsilon}\right)
$$

Using this relationship, we may estimate the gas temperature at arbitrary points from the known solid temperature and inlet gas temperature $t_{a(0)}=t_{0}$.

The integral in Eq. (3I) is written with the notation

$$
S S_{(l)}=\frac{\beta_{n}^{2}}{\beta_{1}^{2}} e^{-\left\langle\beta_{n}^{2} / \beta_{1}^{2}\right) \tau} \int_{0}^{i \Delta \tau} e^{\left(\beta_{n}^{2} / \beta_{1}^{2}\right) \lambda} t_{G} d \lambda
$$

Then we have the recurrence formula on

$$
S S_{(l)}=e^{-\left(\beta_{n}^{2} / \beta_{1}^{2}\right) \Delta \tau} S_{(l-1)}+\left(1-e^{-\left(\beta_{n}^{2} / \beta_{1}^{2}\right) \Delta \tau}\right) t_{G}
$$

Eq.(31) can easily be calculated by Eq.(35), so that we may also evaluate the solid temperature by the known gas temperature.

The outline of the procedure of the numerical method is as follows:

(1) Evaluate successively the gas temperature at initial stage at each point using Eq.(33) with the known initial solid temperature $t_{S(i)}=0$, and the known inlet gas temperature $t_{0}(=1)$;

(2) Evaluate the solid temperature at $\Delta \theta$ at each point by computing the sum of the $(n-1)$ terms of the right side of Eq.(31) by Eq. (35);

(3) Evaluate the gas temperature using again Eq.(33) from the solid temperature obtained in (2) and the known inlet gas temperature $t_{0}(=1)$; and

(4) Repeat the iterative steps (2) and (3) as long as the summation of $\Delta \theta$ becomes the given period of time.

The calculated results in the case of $a h^{\prime}=1,2,3$, 4, 5 are showed in Figs. 1 through 5. The error caused by neglecting the terms after the $n$-th term, and by the finite divided intervals in integral is in full less than 0.01 in these computations.

Table 1 is the numerical table for the calculation of the dimensionless variables.

Figs. 1 through 5 expressed by dimensionless variable are practical and useful for the problem in the region of Biot number 1 to 5 .

\section{Application to the Cooling of Eggs}

Fig. 6 shows the outline of the experimental apparatus. Cool air was drawn by the blower through a column of prewarmed eggs, $A$. The temperatures of the air at the top (inlet) and the bottom (outlet) of the column, and the temperature of the center of an egg at the bottom were measured and recorded with a pen recorder employing three copper-constantan thermocouples, $D$. The flow rate of air was measured by the orifice, $F$.

Fig. 7 shows experimental and theoretical cooling curves for the packed bed of eggs. The following is the comuptation of the theoretical curves using the theory in this paper. The weight of each egg is $56.8 \mathrm{gr}$ on average, and the volume is $51.9 \mathrm{~cm}^{3}$. The external area is estimated from the results of A. L. Romanoff and A. J. Romanoff ${ }^{8}$ as $67.05 \mathrm{~cm}^{2}$. The column of one hundred sixty-six eggs was $50 \mathrm{~cm}$ high in a circular polyvinyl chloride pipe of $20 \mathrm{~cm}$ diameter. The external area per unit volume is 
Fig. I Computed temperature history for value of $a h^{\prime}=1.0$

Fig. 2 Computed temperature history for value of $a h^{\prime}=2.0$

Fig. 3 Computed temperature history for value of $a h^{\prime}=3.0$

Fig. 4 Computed temperature history for value of $a h^{\prime}=4.0$
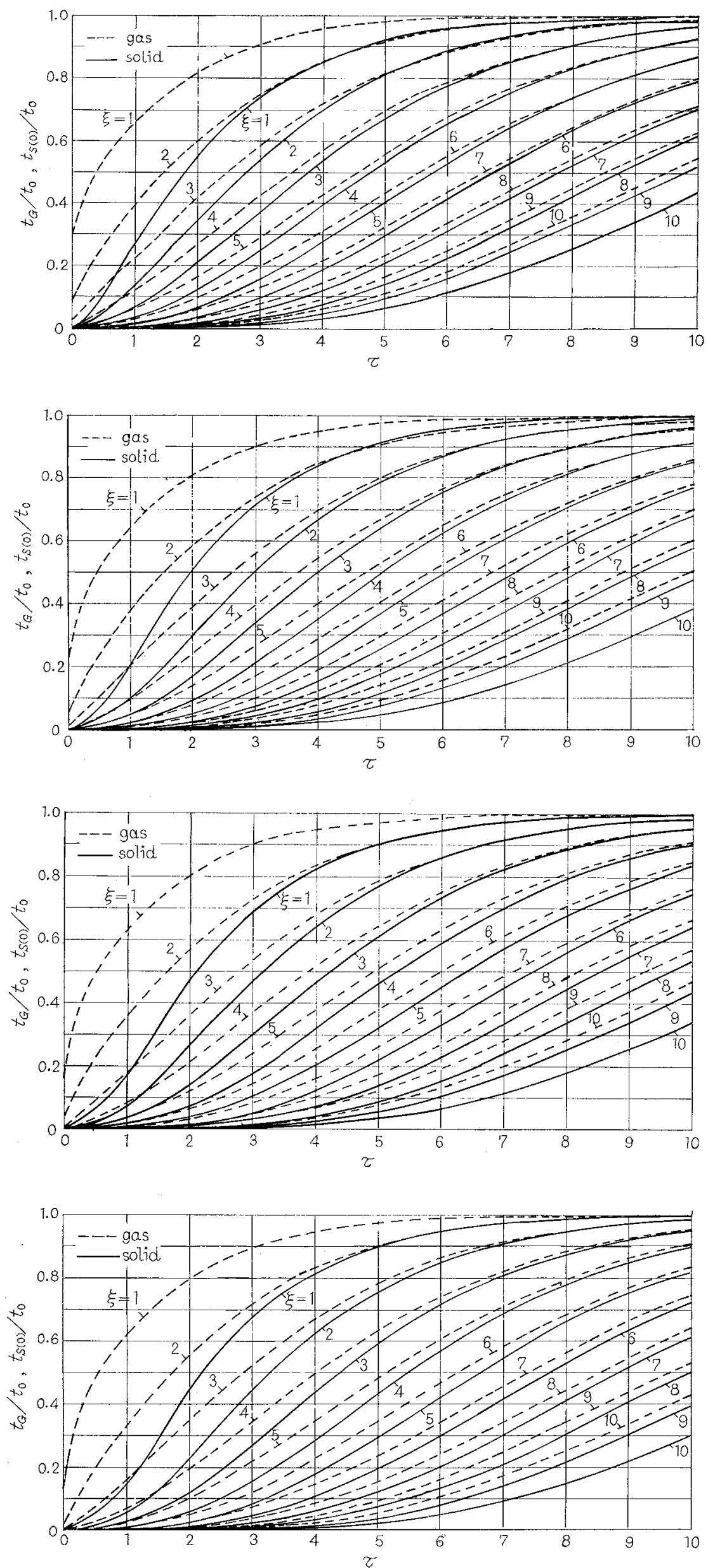

(31) 


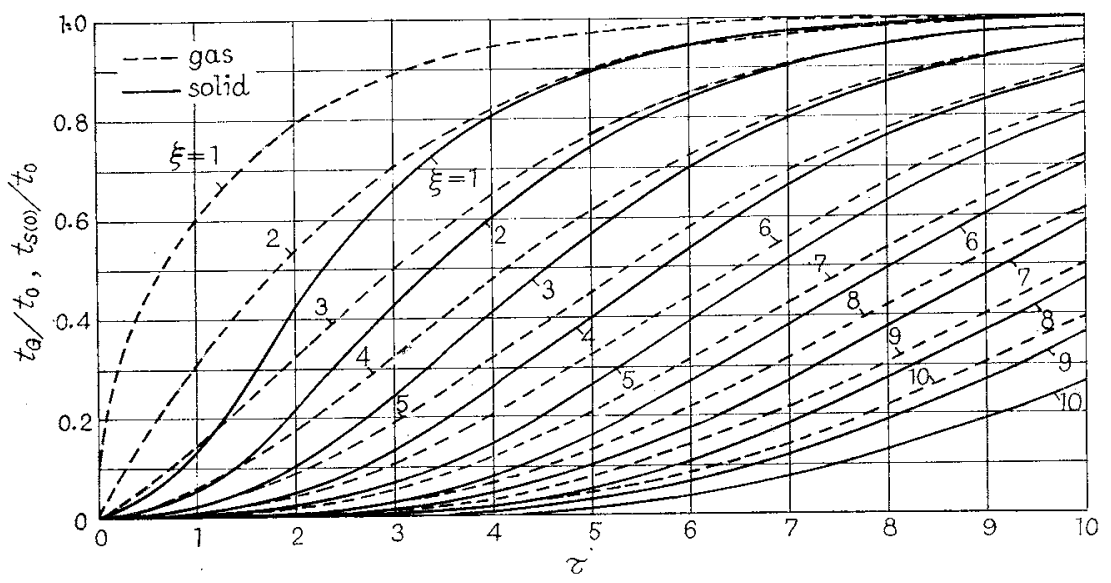

Fig. 5 Computed temperature history for value of $a h^{\prime}=5.0$

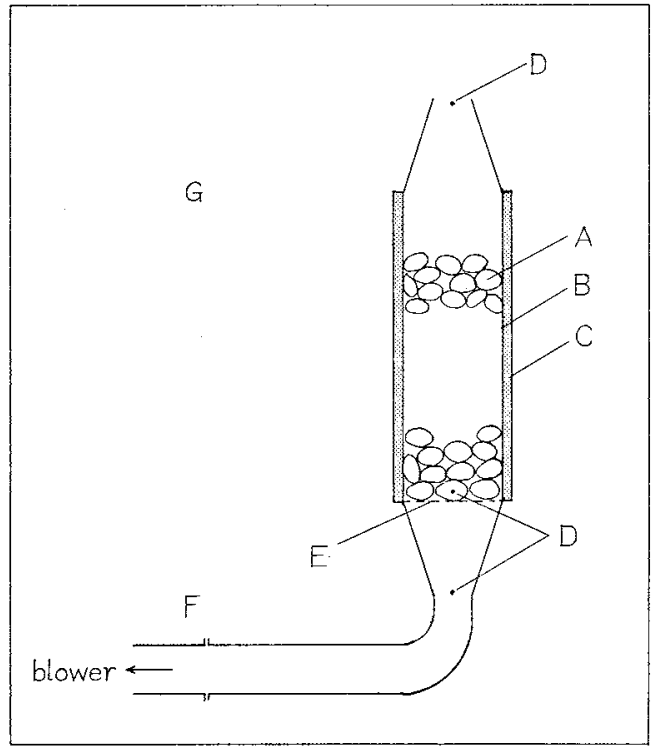

A: Eggs B: Polyvinyl chloride pipe of $20 \mathrm{~cm}$ diameter C: Glass wool D: Thermocouple E: Wire netting F: Orifice G: Constanttemperature room

Fig. 6 Diagram of experimental equipment

\begin{tabular}{cccc}
\multicolumn{3}{l}{$\begin{array}{l}\text { Table } 1 \\
\text { dimensionless variables }\end{array}$} & \multicolumn{3}{l}{ Numerical values used for calculating } \\
$a h^{\prime}$ & $\beta_{1}$ & $\beta_{1}^{2}$ & $\varepsilon$ \\
\hline 0.1 & 0.5423 & 0.2941 & 0.9801 \\
0.2 & 0.7593 & 0.5765 & 0.9603 \\
0.3 & 0.9208 & 0.8478 & 0.9407 \\
0.4 & 1.053 & 1.108 & 0.9213 \\
0.5 & 1.166 & 1.359 & 0.9021 \\
0.6 & 1.264 & 1.599 & 0.8832 \\
0.7 & 1.353 & 1.829 & 0.8646 \\
0.8 & 1.432 & 2.051 & 0.8462 \\
0.9 & 1.504 & 2.263 & 0.8282 \\
1.0 & 1.571 & 2.467 & 0.8106 \\
2.0 & 2.029 & 4.116 & 0.6540 \\
2.82 & 2.251 & 5.066 & 0.5530 \\
3.0 & 2.289 & 5.239 & 0.5338 \\
4.0 & 2.456 & 6.030 & 0.4437 \\
5.0 & 2.570 & 6.607 & 0.3758 \\
6.0 & 2.654 & 7.042 & 0.3240 \\
7.0 & 2.716 & 7.379 & 0.2835 \\
8.0 & 2.765 & 7.647 & 0.2514 \\
9.0 & 2.804 & 7.865 & 0.2254 \\
10.0 & 2.836 & 8.045 & 0.2040 \\
$\infty$ & 3.142 & 9.870 & 0 \\
\hline
\end{tabular}

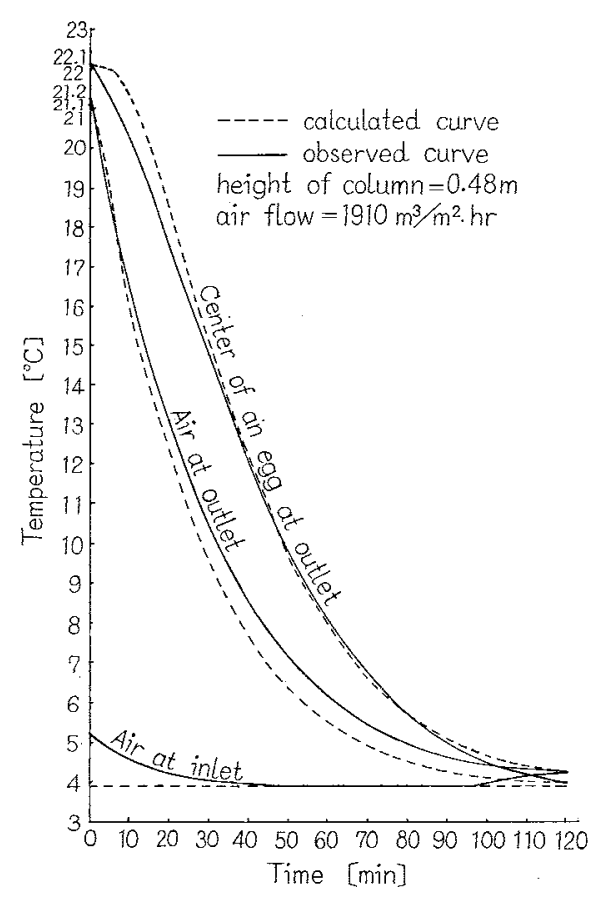

Fig. 7 Observed and calculated cooling curves of air and center of eggs at bottom of column

$a_{Y}=166 \times 67.05 \times 10^{-4} /\left(0.5 \times 0.1^{2} \times 3.142\right)=70.86 \mathrm{~m}^{2} /$ $\mathrm{m}^{3}$. The external void fraction of column was $F_{e}=$ $1-166 \times 51.9 \times 10^{-6} /\left(0.5 \times 0.1^{2} \times 3.142\right)=0.451$. The radius was taken as $23 \mathrm{~mm}$. The specific heat of the egg calculated from Siebel's formula ${ }^{7}$ with the percentage of water was $C_{S}=0.79 \mathrm{kcal} / \mathrm{kg} \cdot{ }^{\circ} \mathrm{C}$. Its thermal diffusivity ${ }^{3)}$ was taken as $4.88 \times 10^{-4} \mathrm{~m}^{2} / \mathrm{hr}$. Its densityis $\rho_{S}=56.8 \times 10^{-3} /\left(51.9 \times 10^{-6}\right)=1090 \mathrm{~kg} / \mathrm{m}^{3}$, and its thermal conductivity is $k=0.422 \mathrm{kcal} / \mathrm{m} \cdot \mathrm{hr} \cdot{ }^{\circ} \mathrm{C}$. The specific heat and density of the air at the mean temperature $13^{\circ} \mathrm{C}$ were taken as $0.24 \mathrm{kcal} / \mathrm{kg} \cdot{ }^{\circ} \mathrm{C}$ and $1.22 \mathrm{~kg} / \mathrm{m}^{3}$.

The superficial veolcity of the air is $u_{M}=1910 \mathrm{~m} / \mathrm{hr}$. The actual average linear velocity in the column then is $u=u_{M} / F_{e}=4235 \mathrm{~m} / \mathrm{hr}$.

The heat transfer coefficient of egg-air was estimated to be $51.8 \mathrm{kcal} / \mathrm{kg} \cdot{ }^{\circ} \mathrm{C}$ by Meek's result ${ }^{1)}$ :

$$
S t=2.06 R e^{-0.42}, R e=900-4000
$$

In nomenclature: $\quad a h^{\prime}=0.023 \times 51.8 / 0.422=2.82$ 
By Table 1: $\quad \varepsilon=0.5530, \beta_{1}^{2}=5.066$

In Eq. (11): $\quad \tau=\left(4.88 \times 10^{-4} \times 5.066 /(0.023)^{2}\right) \theta^{\prime}$ $=4.67 \theta^{\prime} \div 4.67 \theta$

In nomenclature: $k_{2}=51.8 \times 70.86 /(0.24 \times 1.22 \times$ $0.451)=2.78 \times 10^{4} \mathrm{hr}^{-1}$.

In Eq. $(12): \quad \xi=\left(0.5530 \times 2.78 \times 10^{4} / 4235\right) \times 0.48=$ 1.74 .

The observed initial temperature of the egg was at $22.1^{\circ} \mathrm{C}$. This differs from the assumption that the initial temperature of solid is zero, so that the temperature of the preceding article should be expressed in values relative to $22.1^{\circ} \mathrm{C}$. The inlet air temperature was taken as $3.9^{\circ} \mathrm{C}$ in spite of a little variation(Fig. 7). The computed curves, as shown in Figs. 1 through 5, were selected for $a h^{\prime}=2.82$ and $\xi=1.74$. Then the ordinates were read on the curves, and these values were multiplied by $3.9-22.1=-18.2$, and 22.1 was added to these. The dimensionless time was converted to real time using $\theta=0.214 \tau$. The ordinate $t_{S}(0)$ and $t_{G}$ for the abscissa were plotted. These agree well with observed results as shown in Fig. 7.

\section{Appendix}

\section{Derivation of Eq. (19)}

Substituting

into Eq.(18) yields:

$$
t_{G}=e^{-\tau} t
$$

$$
\frac{\partial t}{\partial \xi}=-t+\int_{0}^{t} t d \lambda
$$

and the boundary condition (11) becomes

$$
t=t_{0} e^{\tau}
$$

Taking the Laplace transform of Eq.(A2) with respect to $\tau$ and letting

$$
F=L_{\tau}[t]
$$

yeilds :

$$
\frac{\partial F}{\partial \xi}=-F+\frac{1}{s} F
$$

Eq.(A5) has the solution

$$
F=C e^{\left(-1+\frac{1}{s}\right) \xi}
$$

Taking the Laplace transform of Eq.(A3) at 0 yeilds:

$$
F=\frac{t_{0}}{s-1}
$$

Substituting $\xi=0$ into Eq.(A6), and using Eq.(A7), we get

$$
C=\frac{t_{0}}{s-1}
$$

Substituting Eq.(A8) into Eq.(A6) yields:

$$
F=\frac{t_{0}}{s-1} e^{\left(-1+\frac{1}{s}\right) \xi}
$$

Eq.(A9) is modified to

$$
F=t_{0} e^{-\xi}\left(\frac{1}{s-1}\left(e^{\frac{1}{s} \xi}-1\right)+-\frac{1}{s-1}\right)
$$

Applying the known formulas ${ }^{5,6}$ )

$$
\begin{gathered}
L_{\tau}\left[e^{\tau}\right]=\frac{1}{s-1} \\
L_{\tau}\left(\sqrt{\frac{\nu}{\tau}} I_{1}(2 \sqrt{\nu \tau})\right)=e^{-\frac{\nu}{s}}-1
\end{gathered}
$$

and the convolution theorem, we obtain

$$
t=t_{0} e^{-\xi}\left(\int_{0}^{\tau} e^{(\tau-\lambda)} \sqrt{\frac{\xi}{\lambda}} I_{1}(2 \sqrt{\xi \lambda}) d \lambda+e^{\tau}\right)
$$

VOL. 4 NO. $2 \quad 1971$

$$
=t_{0} e^{-\xi+\tau}\left(e^{-\tau} I_{0}(2 \sqrt{\xi \tau})+\int_{0}^{t} e^{-\lambda} I_{0}(2 \sqrt{\xi \lambda}) d \lambda\right)
$$

Then we have

$$
t_{G} \mid t_{0}=e^{-\tau} t / t_{0}=e^{-\tau} I_{0}(2 \sqrt{\xi \tau})+\int_{0}^{\tau} e^{-\xi-\lambda} I_{0}(2 \sqrt{\xi \lambda}) d \lambda
$$

Reduction of Eq. $(10)^{\prime}$ to Schumann's equation in infinite thermal diffusivity of solid

All terms after the first vanish because $a h^{\prime}$ reduces to zero, although $a \alpha_{n}$ does not reduce to zero. Consequently, using Eq.(22), Eq. (10)' is reduced to

$$
t_{S}(a)=t_{G}-\left(t_{G}-\kappa \alpha_{1}^{2} e^{-\kappa \alpha_{1}^{2} \theta^{\prime}} \int_{0}^{\theta^{\prime}} e^{\kappa \sim_{1}^{\prime} \lambda} t_{G} d \lambda\right)
$$

Multiplying both sides of Eq.(B1) by

$$
e^{r \alpha_{1}^{2} \theta^{\prime}}
$$

we have

$$
t_{S}(a) e^{r \alpha_{1}^{2} \theta^{\prime}}=\kappa \alpha_{1}^{2} \int_{0}^{\theta \prime} e^{r \alpha_{1}^{2} \lambda} t_{\sigma^{\prime}} d \lambda
$$

Differnetiating both sides of Eq. (B2) with respect to $\theta^{\prime}$ we have

$$
\frac{\partial t_{S}(a)}{\partial \theta^{\prime}}=\kappa \alpha_{1}^{2}\left(t_{G}-t_{S}(a)\right)
$$

Considering Eq.(21), we find Eq.(B3) is identical with Eq.(6).

\section{Acknowledgement}

I would like to thank Professor Y. Chuma for his valuable discussions on the present work, and Mr. Y. Hori for his assistance.

\section{Nomenclature}

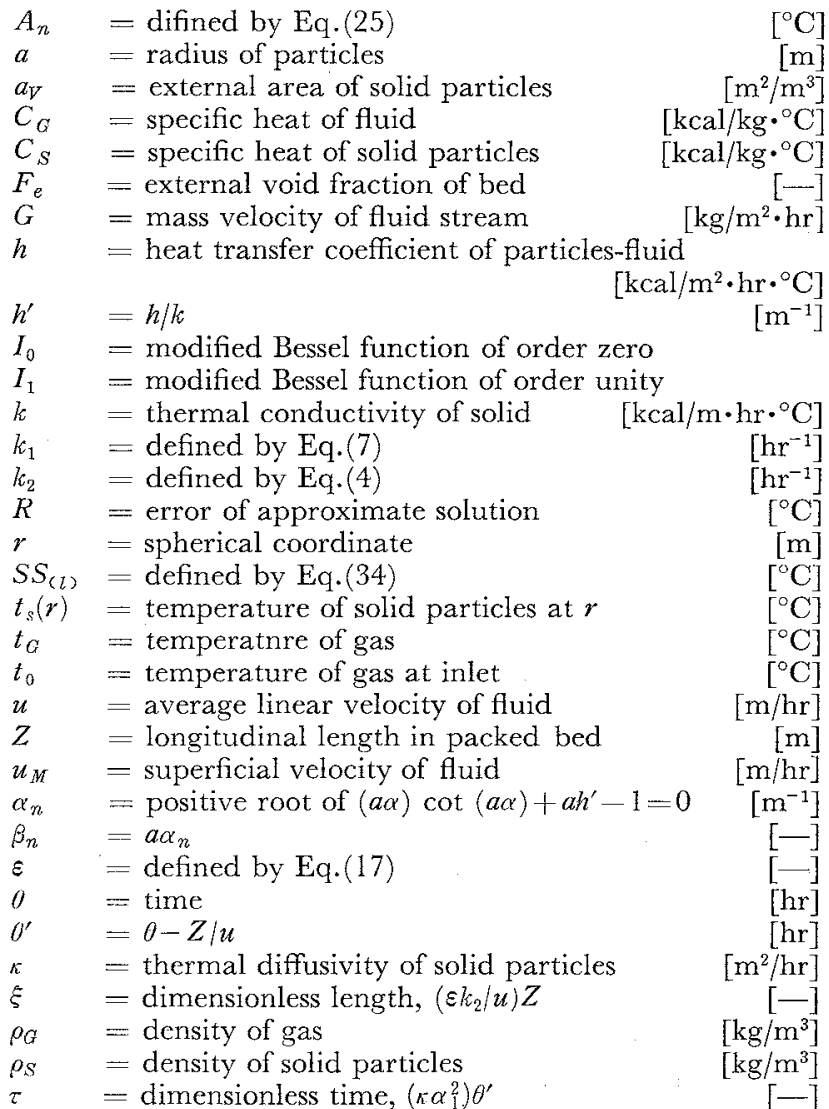

\section{Literature cited}

1) Barker, J. J.: Ind. Eng. Chem., 57, 43(1965)

2) Carslaw, H.S. and J. C. Jaeger: "Conduction of Heat in Solids", p. 31, Oxford Univ. Press, London (1959)

3) Chuma, Y., S. Murata, and S. Uchida: J. Soc. Agric. Machinery, Japan, 30, 100 (1968) . 
4) Furnas, C. C.: Ind. Eng. Chem., 22, 721 (1930)

5) Kondo, J.: "Enzanshi-hō (Operational Calculus)" p. 17, Baifükan, Tokyo (1956)

6) ibid., 205

7) Richey, C. B.: "Agricultural Engineers' Handbook", p. 746, McGraw-Hill, New York (1961)
8) Romanoff, A. L. and A. J. Romanoff: "The Avian Egg", p. 105, John Wiley \& Sons, New York (1949)

9) Schumann, T. E. W.: J. Franklin Inst., 208, 405 (1929)

10) Sugiyama, Y. and K. Nagasaka: Kagaku Koggaku, 22, $547(1958)$

\title{
SIGNIFICANCE OF SENSIBLE HEATS ON NONISOTHERMAL MOVING BOUNDARY ANALYSIS OF SOLID-GAS REACTIONS*
}

\author{
MASARU ISHIDA, RICHARD C. BAILIE** AND TAKASHI SHIRAI \\ Research Laboratory of Resources Utilization, Tokyo Institute of Technol- \\ ogy, Tokyo, Japan
}

\begin{abstract}
Moving boundary problems with mass and heat transfer are discussed and an apparent thermal conductivity $k_{e}^{\prime}$ is proposed to represent the effect of the sensible heat of the diffusing gas on the moving rate of the boundary. Although this effect is usually small, it should be taken into account for some cases when the drying of wet porous bodies or the thermal decomposition of solids is conducted at a high ambient temperature.
\end{abstract}

\section{Introduction}

The melting of ice and the thermal decomposition of solid are usually treated as moving boundary problems. In each case, the heat is transmitted through the product layer to the inner moving boundary at which melting or decomposition is taking place.

In the former process, because the density of water and that of ice are close, the product water remains near the place where it existed as ice before melting. On the other hand, the latter process includes a gas phase and because the density of gas is much smaller than that of solid, the product gas diffuses outward through the product solid.

For endothermic reactions, the gas produced at the decomposition surface must be heated up from the decomposition temperature to the temperature of the outer surface while it diffuses through the solid product. Accordingly, some quantity of heat supplied from the outer surface must be consumed in the sensible heat of the product gas as well as in the sensible heat of the product solid ${ }^{6}$. The drying of wet porous bodies during the falling-rate period and some solid-gas reactions represent similar processes with simultaneous heat and mass transfer, ${ }^{4}$ ).

The effect of the sensible heat of diffusing gas on the heat transfer coefficient has been analyzed by Ackerman ${ }^{13}$ and Colburn and Drew ${ }^{3)}$. However, such effect on the moving boundary problem has not yet been studied.

* Received on September 19, 1970

* Department of Chemical Engineering, West Virginia University, Morgantown, W. Va., U.S.A.
The purpose of this study is to examine the data in previous work and evaluate the effect on moving boundary problems.

\section{Derivation of Equations}

\section{1-1. Basic equations of the problem}

Consider a solid-gas reaction represented by the following equation:

$$
S_{(s)}+a A_{(g)} \longrightarrow S_{(s)}^{\prime}+b B_{(g)}
$$

where $a$ is considered to be zero for the thermal decomposition of solids or for the drying of porous bodies. Assume:

1) An infinite slab of thickness $2 Z$, which remains constant during the reaction.

2) The temperature at the outer surface $T_{0}$ and the reaction surface $T_{c}$ remain constant.

Fig. 1 illustrates the above system schematically. The basic equation for the temperature profile within the product layer is

$$
\begin{aligned}
& C_{S^{\prime}} c_{p S^{\prime}}(\partial T / \partial t)=k_{\theta}\left(\partial^{2} T / \partial z^{2}\right) \\
& +C_{S o}\left(d z_{c} / d t\right)\left(b c_{p_{B}}-a c_{p A}\right)(\partial T / \partial z)
\end{aligned}
$$

The first term on the left-hand side and the second term on the right-hand side correspond to the sensible heats of product solid and diffusing gas, respectively.

The boundary conditions are:

$$
\begin{array}{ll}
\begin{array}{l}
T=T_{o} \\
T=T_{c}
\end{array} & \text { at } z=0 \\
-\bar{c}_{e}(\partial T / \partial z)=C_{S} \Delta H_{S}\left(d z_{c} / d t\right) & \text { at } z=z_{c} \\
z_{c}=0 & \text { at } t=0
\end{array}
$$

The third boundary condition indicates that at the reaction surface, $Z=Z_{v}$, the rate of heat conduction 